BMJ Open Diabetes

Research \& Care

\section{Association of renin and aldosterone with glucose metabolism in a Western European population: the KORA F4/ FF4 study}

Cornelia Then (D) , ${ }^{1,2}$ Katrin Ritzel, ${ }^{1}$ Christian Herder (D) , ${ }^{3,4,5}$ Holger Then, ${ }^{6}$ Chaterina Sujana, ${ }^{2,7,8}$ Margit Heier, ${ }^{7,9}$ Christa Meisinger, ${ }^{10,11}$ Annette Peters, ${ }^{2,7,12}$ Wolfgang Koenig, ${ }^{12,13,14}$ Wolfgang Rathmann, ${ }^{3,15}$ Michael Roden, $3,4,5$ Haifa Maalmi, ${ }^{3,5}$ Michael Stumvoll, ${ }^{16}$ Thomas Meitinger, ${ }^{12,17}$ Martin Bidlingmaier, ${ }^{1}$ Jochen Seissler, ${ }^{1,2}$ Barbara Thorand (D) ,2,7 Martin Reincke ${ }^{1}$
To cite: Then C, Ritzel K, Herder C, et al. Association of renin and aldosterone with glucose metabolism in a Western European population: the KORA F4/ FF4 study. BMJ Open Diab Res Care 2022;10:e002558. doi:10.1136/

bmjdrc-2021-002558

- Additional supplemental material is published online only. To view, please visit the journal online (http://dx.doi. org/10.1136/bmjdrc-2021002558).

BT and MRe contributed equally.

Received 24 August 2021 Accepted 26 December 2021

Check for updates

(c) Author(s) (or their employer(s)) 2022. Re-use permitted under CC BY-NC. No commercial re-use. See rights and permissions. Published by BMJ.

For numbered affiliations see end of article.

Correspondence to Dr Cornelia Then; cornelia.then@med.unimuenchen.de

\section{ABSTRACT}

Introduction Primary aldosteronism is associated with impaired glucose tolerance. Whether plasma aldosterone and/or renin concentrations are associated with type 2 diabetes and continuous measures of glucose metabolism in the general population is still under debate.

Research design and methods The analyses included 2931 participants of the KORA F4 study at baseline and 2010 participants of the KORA FF4 study after a median follow-up of 6.5 years. The associations of active plasma renin and aldosterone concentrations with type 2 diabetes and continuous measures of glucose metabolism were assessed using logistic and linear regression models. Results were adjusted for sex, age, body mass index (BMI), estimated glomerular filtration rate, potassium, use of ACE inhibitors, angiotensin receptor blockers, beta blockers, diuretics and calcium channel blockers.

Results Cross-sectionally, renin was associated with type 2 diabetes (OR per SD: $1.25,95 \% \mathrm{Cl} 1.10$ to $1.43, \mathrm{p}<0.001$ ), fasting glucose, 2-hour glucose, insulin, proinsulin, HOMA-B (homeostasis model assessment of beta cell function) and HOMA-IR (homeostasis model assessment of insulin resistance) (all $p$ values $<0.001$ ). Aldosterone was not associated with type 2 diabetes (OR: 1.04, 95\% Cl 0.91 to 1.19; $p=0.547$ ) but with insulin, proinsulin and HOMA-IR (all $p$ values $<0.001)$. The aldosterone-renin ratio was inversely associated with type 2 diabetes and several measures of glucose metabolism. Longitudinally, neither renin (OR: 1.12, $95 \% \mathrm{Cl} 0.92$ to 1.36 ) nor aldosterone (OR: $0.91,95 \% \mathrm{Cl}$ 0.74 to 1.11) were associated with incident type 2 diabetes. Renin was inversely associated with changes of insulin concentrations.

Conclusions In the KORA F4/FF4 study, renin and aldosterone were not associated with incident type 2 diabetes and largely unrelated to changes of measures of glucose metabolism. Cross-sectionally, aldosterone was associated with surrogate parameters of insulin resistance. However, these associations were not independent of renin.

\section{INTRODUCTION}

Increasing evidence indicates a bidirectional interplay of aldosterone with metabolic

\section{Significance of this study}

What is already known about this subject?

- Elevated aldosterone concentrations in primary aldosteronism are associated with impaired glucose tolerance and type 2 diabetes. The underlying mechanisms remain elusive, and the association of aldosterone and renin with glucose metabolism in the general population is still under debate.

\section{What are the new findings?}

- In a Western European general population, renin was associated with prevalent type 2 diabetes, continuous measures of glucose metabolism and the adipokines leptin and retinol-binding protein-4.

- Aldosterone was cross-sectionally associated with insulin resistance and leptin. These associations were not independent of renin.

> Neither renin nor aldosterone were longitudinally positively associated with incident type 2 diabetes or changes in continuous measures of glucose metabolism.

How might these results change the focus of research or clinical practice?

- Elevated renin and aldosterone are cross-sectionally associated with impaired glucose tolerance. However, no longitudinal associations with measures of glucose metabolism were detectable, indicating that moderately elevated aldosterone concentrations do not seem to play a major role for the development of impaired glucose metabolism in the general Western European population. Future research should focus on possible ethnic differences of the relation of aldosterone with type 2 diabetes.

alterations. Obesity may promote idiopathic hyperaldosteronism via secretory products of adipocytes stimulating mineralocorticoid secretion. ${ }^{12}$ Vice versa, overactivation of the mineralocorticoid receptor plays a role in 
the pathophysiology of metabolic alterations, including dysglycemia. ${ }^{3}$

Preclinical data indicate an interference of aldosterone with both insulin secretion and insulin resistance. In mice, a high-fat diet leads to dysregulation of the renin-angiotensin-aldosterone system (RAAS), ${ }^{4}$ and aldosterone excess decreases glucose-stimulated insulin secretion. ${ }^{5}$ In rat skeletal muscle, elevated aldosterone inhibits the expression of the insulin receptor and the translocation of glucose transporter-4 to the plasma membrane, resulting in decreased glucose uptake. ${ }^{6}$ The effects of aldosterone on metabolism may partly be mediated by subclinical inflammatory responses and alterations in adipokine expression. ${ }^{7}$

In humans, the association of elevated aldosterone with alterations of glucose metabolism has mainly been studied in the setting of primary aldosteronism. ${ }^{8}$ Persons with primary aldosteronism are more likely to have type 2 diabetes (T2D) compared with the general population, ${ }^{9}$ and the aldosterone-to-renin ratio (ARR) within primary aldosteronism is higher in case of concomitant diabetes. ${ }^{10}$ Longitudinal data suggest a causal role of primary aldosteronism for impaired glucose tolerance (IGT), since adrenalectomy reduced glucose and insulin values, ${ }^{11}$ improved insulin sensitivity, ${ }^{12}{ }^{13}$ and restored a decreased first-phase insulin secretion in an intravenous glucose tolerance test ${ }^{14}$ as well as the insulin response to a hyperglycemic clamp. ${ }^{15}$ However, the findings for insulin sensitivity remain inconsistent, since Adler et $a l^{15}$ found a decreased insulin sensitivity after treatment with adrenalectomy or mineralocorticoid receptor antagonists.

Beyond primary aldosteronism, data from other populations support a relationship of aldosterone with glucometabolic alterations. Plasma aldosterone concentrations were associated with insulin resistance in patients with heart failure, ${ }^{16}$ in HIV-infected patients $^{17} 18$ and in normotensive individuals. ${ }^{19}$ In general population-based settings, aldosterone was associated with the metabolic syndrome in two German populations ${ }^{20}$ and with insulin resistance in men but not in women in an African-American population. ${ }^{21}$ Longitudinally, baseline plasma aldosterone was associated with the development of insulin resistance in a Japanese general population, ${ }^{22}$ renin and aldosterone were associated with incident T2D in an AfricanAmerican population, ${ }^{23}$ and the multiethnic study of atherosclerosis found an association of aldosterone, but not renin, with incident T2D. Of note, in the latter study, the association was stronger in Chinese and African-Americans as compared with Hispanic and non-Hispanic white Americans. ${ }^{24}$

The aim of the present study was to determine the cross-sectional and longitudinal associations of renin and aldosterone with pre-diabetes, T2D and continuous measures of glucose metabolism in a large Western European population of mainly Caucasian ethnicity. In light of preliminary data indicating a relation of the RAAS with adipokines, ${ }^{17}$ we additionally examined the cross-sectional associations of the RAAS parameters with selected adipokines.

\section{METHODS}

\section{Study participants and definition of variables}

The KORA (Cooperative Health Research in the Region of Augsburg) F4 (2006-2008) and FF4 (2013-2014) studies are follow-up examinations of the populationbased KORA S4 study (1999-2001). All participants gave written informed consent. Recruitment and eligibility criteria for the KORA studies, study design, standardized sampling methods and data collection (medical history, medication, anthropometric and blood pressure measurements) have been described elsewhere. $^{2526}$

Participants with a diabetes type other than type 2 or unknown glucose tolerance were excluded from the analyses $(n=75)$. In the cross-sectional analysis, all variables necessary for the analysis of the association of renin and aldosterone with T2D were available in 2931 (out of 3080) participants. Measurements of glucose metabolism and adipokines were available in 1093-2743 participants depending on the outcome (online supplemental figure 1). Total proinsulin was measured in the first 1567 participants of the KORA F4 study. Adiponectin and omentin-1 were measured in participants aged $\geq 62$ years.

Of the 2931 participants, 154 died before the follow-up examination and 697 declined participation in the FF4 survey or could not be contacted. The study sample in the longitudinal F4/FF4 examination comprised 1834 participants for the analysis of incident T2D. Participants with missing data for the continuous outcomes were excluded from the respective analyses. The numbers of participants included in each analysis are given in the results tables and illustrated in online supplemental figure 1. The median (first; third quartile) follow-up time was 6.5 (95\% CI 6.3 to 6.6$)$ years.

Criteria for clinically diagnosed diabetes mellitus were a validated medical diagnosis or current self-reported use of glucose-lowering agents. After an overnight fasting period, all participants without clinically diagnosed diabetes underwent a standard $75 \mathrm{~g}$ oral glucose tolerance test. Newly diagnosed diabetes, IGT, impaired fasting glucose (IFG), and normal glucose tolerance (NGT) were defined according to the 1999 WHO diagnostic criteria based on both fasting and postchallenge glucose values (T2D: $\geq 7.0 \mathrm{mmol} / \mathrm{L}$ fasting and $/$ or $\geq 11.1 \mathrm{mmol} / \mathrm{L}$ 2-hour glucose; IFG: $\geq 6.1 \mathrm{mmol} / \mathrm{L}$ and $<7.0 \mathrm{mmol} / \mathrm{L}$ fasting glucose; IGT: $\geq 7.8$ and $<11.1 \mathrm{mmol} / \mathrm{L}$ 2-hour glucose). Pre-diabetes was defined as IFG and/or IGT. Incident pre-diabetes/T2D was defined as progression from NGT to pre-diabetes $(n=245)$ or T2D $(n=38)$. Incident T2D ( $\mathrm{n}=130)$ was defined as progression from NGT or pre-diabetes to T2D.

Arterial hypertension was defined as a systolic blood pressure $\geq 140 \mathrm{~mm} \mathrm{Hg}$ and/or a diastolic blood pressure $\geq 90 \mathrm{~mm} \mathrm{Hg}$ (mean values of the second and third 
of three blood pressure measurements), and/or use of antihypertensive medication, given that the participants were aware of being hypertensive. The methods for 'study participants and definition of variables' are similar to those previously described. ${ }^{27}$

\section{Laboratory measurements}

Blood samples were collected after an overnight fast of at least 8 hours in sitting position after a rest of 10 min (sitting) and were kept at room temperature until centrifugation. Plasma was separated immediately, serum after $30 \mathrm{~min}$. Samples were assayed immediately or stored at $-80^{\circ} \mathrm{C}$. Plasma renin concentrations were measured using the Liaison active renin assay (Diasorin, Dietzenbach, Germany) using monoclonal antibodies to only detect active renin molecules without interference with prorenin. Intra-assay and interassay coefficients of variation were below $5.6 \%$ and $12.2 \%$, respectively, and the functional sensitivity was $<2.0 \mu \mathrm{U} /$ $\mathrm{mL}$. Plasma aldosterone concentrations were measured with an inhouse immunoflurometric assay as described previously. ${ }^{28}$ Interassay and intra-assay coefficients of variation were $15.2 \%$ and $7.3 \%$ in low, and $8.0 \%$ and $4.4 \%$ in high concentrations, respectively. Serum creatinine was determined with a modified Jaffe test (Krea Flex; Dade Behring, Deerfield, Illinois, USA). Glucose levels were measured in serum using a hexokinase method (GLUFlex, Dade Behring) at baseline and an enzymatic colorimetric method on a Dimension Vista 1500 instrument (Siemens) or the GLUC3 assay on a Cobas c702 instrument (Roche Diagnostics, Mannheim, Germany) at follow-up. ${ }^{29}$ Hemoglobin A1c (HbA1c) was measured in hemolyzed whole blood using the cationexchange high performance liquid chromatographic, photometric VARIANT II TURBO HbAlc Kit - 2.0 assay on a VARIANT II TURBO Hemoglobin Testing System (Bio-Rad Laboratories Inc, Hercules, USA). Insulin was measured by an electrochemiluminescence immunoassay on a Cobas e602 instrument (Roche) in KORA F4. KORA FF4 serum insulin concentrations were assessed by a solid-phase enzyme-labeled chemiluminescent immunometric assay on an Immulite 2000 systems analyzer (Siemens) or by an electrochemiluminescence immunoassay on a Cobas e602 instrument (Roche). Insulin values were calibrated to the Roche assay as described previously. ${ }^{26}$ Intact proinsulin (frozen EDTA plasma) was measured by ELISA (intact human proinsulin ELISA, Cat.-No. EZHIPI-17K, Linco Research, St Charles, Missouri, USA). Chemerin serum concentrations were determined using a commercially available ELISA kit (Human Chemerin ELISA, Biovendor, Heidelberg, Germany) with a sensitivity of $0.1 \mathrm{ng} / \mathrm{mL}$ and intra-assay and interassay coefficients of variance of $6.0 \%$ and $7.6 \% .^{30}$ Plasma concentrations of retinol-binding protein- 4 were measured by immunonephelometry using a BN II analyzer. The intra-assay and inter-assay coefficients of variation were $<10 \%{ }^{31}$ Leptin was measured by ELISA (Mercodia, Uppsala,
Sweden) with intra-assay and interassay coefficients of variation of $<10 \%$. Serum concentrations of omentin-1 and adiponectin were measured using the Human Omentin-1 ELISA (BioVendor, Brno, Czech Republic) and the Human Total Adiponectin/Acrp30 Quantikine ELISA Kit (R\&D Systems) with intra-assay coefficients of variation (CVs) of $2.0 \%$ and $4.0 \%$, respectively, and interassay CVs of $3.8 \%$ and $8.0 \%$, respectively. ${ }^{32}$ Progranulin serum concentrations were determined using the Progranulin human ELISA Kit AdipoGen from AdipoGen (Seoul, Korea). ${ }^{30}$ Serum vaspin concentrations were quantified using a commercial ELISA kit (AdipoGen) with a sensitivity of $12 \mathrm{pg} / \mathrm{mL}$. The intra-assay and interassay coefficients of variance were $1.3 \%-3.8 \%$ and $3.3 \%-9.1 \%$, respectively. ${ }^{30}$

Estimated glomerular filtration rate (eGFR) was calculated using the Chronic Kidney Disease Epidemiology Collaboration equation (2009) based on serum creatinine. ${ }^{33}$ Homeostasis model assessment of insulin resistance (HOMA-IR: fasting insulin (in $\mathrm{pmol} / \mathrm{L}$ ) $\mathrm{x}$ fasting glucose (in mmol/L) $\div 135$ ), homeostasis model assessment of beta cell function (HOMA-B: fasting insulin $(\mu \mathrm{U} / \mathrm{mL}) \times 20 \div($ glucose $(\mathrm{mmol} / \mathrm{L})-$ $3.5) \%$ ) were calculated in all participants except those with antihyperglycemic treatment.

\section{Statistical analyses}

Characteristics of the study participants were compared between participants without and with T2D using t-tests in case of approximately normally distributed variables. Mann-Whitney U-tests were performed for variables with skewed distributions. Binomial proportions were compared with $\chi^{2}$ tests. The associations of renin, aldosterone and the ARR with pre-diabetes and T2D were assessed in logistic regression models. The associations with continuous dependent variables were examined in linear regression models. Continuous variables were transformed to a Gaussian distribution by probability integral transformation followed by inverse transform sampling, $z$-standardized and used in calculations per one SD increase. In multivariable logistic and linear regression analyses, the associations were adjusted for sex, age, BMI, arterial hypertension, eGFR, potassium, use of ACE inhibitors, angiotensin receptor blockers, beta blockers, diuretics and calcium channel blockers. For sensitivity analyses, the calculations were repeated after exclusion of participants taking ACE inhibitors, angiotensin receptor blockers, beta blockers, diuretics and calcium channel blockers (online supplemental tables 1-4 and 6). Participants taking glucose-lowering medication were excluded from the analyses involving continuous measures of glucose metabolism. For the longitudinal associations, the deltas of the continuous measures of glucose metabolism (follow-up value (KORA FF4) - baseline value (KORA F4)) were used as outcome variables. The level of statistical significance was set at 5\% (two sided). Bonferroni correction for multiple testing was used (numbers of tests are given in the respective tables). The calculations 
Table 1 Characteristics of the study participants at KOFA F4, overall and stratified by type 2 diabetes at baseline*

\begin{tabular}{|c|c|c|c|c|c|c|c|}
\hline & $\mathbf{N}$ & Total study cohort & $\mathbf{N}$ & No diabetes $\dagger$ & $\mathbf{N}$ & Type 2 diabetes $\ddagger$ & $P$ value $\S$ \\
\hline Male sex, n (\%) & 2931 & $1418(48)$ & 2598 & $1220(47)$ & 333 & $198(59)$ & $<0.0019$ \\
\hline Age (years) & 2931 & $56.2 \pm 13.2$ & 2598 & $54.8 \pm 12.9$ & 333 & $67.0 \pm 9.3$ & $<0.001^{\star \star}$ \\
\hline $\operatorname{BMI}\left(\mathrm{kg} / \mathrm{m}^{2}\right)$ & 2931 & $27.6 \pm 4.8$ & 2598 & $27.2 \pm 4.6$ & 333 & $31.2 \pm 5.1$ & $<0.001^{\star \star}$ \\
\hline eGFR $\left(\mathrm{mL} / \mathrm{min} / 1.73 \mathrm{~m}^{2}\right)$ & 2931 & $89.3(77.6 ; 100.0)$ & 2598 & $90.6(78.9 ; 101.2)$ & 333 & $78.9(64.7 ; 89.1)$ & $<0.001 \dagger \dagger$ \\
\hline Arterial hypertension, $\mathrm{n}(\%)$ & 2931 & $1122(38)$ & 2598 & $859(33)$ & 333 & $263(79)$ & $<0.001 \emptyset$ \\
\hline Pre-diabetes, n (\%) & 2931 & $488(16.6)$ & 2598 & $488(18.8)$ & - & - & - \\
\hline 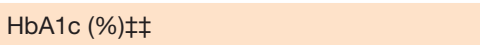 & 2764 & $5.4(5.2 ; 5.7)$ & 2598 & $5.4(5.2 ; 5.6)$ & 333 & $6.1(5.8 ; 6.6)$ & $<0.001 \dagger \dagger$ \\
\hline $\mathrm{HbA} 1 \mathrm{c}(\mathrm{mmol} / \mathrm{mol}) \neq \ddagger$ & 2764 & $35.5(33.3 ; 38.8)$ & 2598 & $35.5(33.3 ; 37.7)$ & 333 & $43.2(39.9 ; 48.6)$ & $<0.001+\dagger$ \\
\hline Fasting glucose $(\mathrm{mg} / \mathrm{dL}) \neq \ddagger$ & 2764 & $93(88 ; 101)$ & 2598 & $93(87 ; 100)$ & $166+†$ & $122(109 ; 135)$ & $<0.001+\dagger$ \\
\hline Fasting glucose (mmol/L) $\ddagger \ddagger$ & 2764 & $5.16(4.88 ; 5.61)$ & 2598 & $5.16(4.82 ; 5.55)$ & $166+\dagger$ & $6.77(6.07 ; 7.49)$ & $<0.001+\dagger$ \\
\hline 2-hour glucose (mg/dL) & 2710 & $104(87 ; 128)$ & 2598 & $103(86 ; 125)$ & 112 & $214(200 ; 241)$ & $<0.001+\dagger$ \\
\hline 2-hour glucose (mmol/L) & 2710 & $5.77(4.77 ; 6.92)$ & 2598 & $5.72(4.77 ; 6.92)$ & 112 & $11.88(11.11 ; 13.36)$ & $<0.001 \dagger \dagger$ \\
\hline 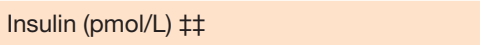 & 2743 & $52.2(37.2 ; 78.0)$ & 2579 & $51.0(36.6 ; 72.0)$ & $164 \dagger \dagger$ & $96(66.0 ; 138.0)$ & $<0.001+\dagger$ \\
\hline Proinsulin (pmol/L) $\neq \ddagger$ & 1438 & $2.9(1.9 ; 4.5)$ & 1356 & $2.8(1.9 ; 4.2)$ & $82+\dagger$ & $6.9(4.8 ; 10.1)$ & $<0.001+\dagger$ \\
\hline Proinsulin-to-insulin ratio $\ddagger \ddagger$ & 1438 & $0.05(0.04 ; 0.08)$ & 1356 & $0.05(0.04 ; 0.07)$ & $82 †+$ & $0.07(0.05 ; 0.103)$ & $<0.001 \dagger \dagger$ \\
\hline HOMA-B $\neq \ddagger$ & 2743 & $104.5(76.9 ; 143.9)$ & 2579 & $104.9(77.9 ; 143.7)$ & $164 \uparrow \dagger$ & $95.6(69.9 ; 150.9)$ & $0.179+\dagger$ \\
\hline 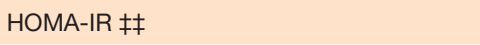 & 2743 & $2.02(1.37 ; 3.08)$ & 2579 & $1.95(1.34 ; 2.88)$ & $164 \uparrow \dagger$ & $5.12(3.29 ; 7.22)$ & $<0.001+\dagger$ \\
\hline Leptin (ng/mL) & 2922 & $12.0(5.3 ; 24.8)$ & 2592 & $11.3(5.0 ; 23.3)$ & 330 & $20.3(9.7 ; 41.5)$ & $<0.001 \dagger \dagger$ \\
\hline Retinol-binding protein-4 (g/L) & 2922 & $0.042(0.036 ; 0.049)$ & 2592 & $0.042(0.035 ; 0.049)$ & 330 & $0.045(0.039 ; 0.053)$ & $<0.001+\dagger$ \\
\hline Adiponectin $(\mu \mathrm{g} / \mathrm{mL})$ & 1093 & $10.11(6.57 ; 15.14)$ & 862 & $10.78(7,21 ; 16.01)$ & 231 & $7.58(5.32 ; 11.11)$ & $<0.001 \dagger \dagger$ \\
\hline Omentin-1 (ng/mL) & 1093 & $489.0(403.6 ; 580.8)$ & 862 & $491.7(406.6 ; 582.5)$ & 231 & $480.8(391.5 ; 576.6)$ & $0.233+\dagger$ \\
\hline Chemerin $(\mathrm{ng} / \mathrm{mL})$ & 1702 & $167.2(135.2 ; 199.6)$ & 1461 & $165.9(133.2 ; 197.8)$ & 241 & $175.7(145.6 ; 216.6)$ & $<0.001 \dagger \dagger$ \\
\hline Progranulin (ng/mL) & 1702 & $137.3(106.4 ; 176.5)$ & 1461 & $136.0(106.2 ; 175.6)$ & 241 & $146.4(109.7 ; 191.1)$ & $0.084 \dagger \dagger$ \\
\hline Vaspin $(\mathrm{ng} / \mathrm{mL})$ & 1702 & $0.58(0.32 ; 1.08)$ & 1461 & $0.57(0.31 ; 1.07)$ & 241 & $0.69(0.39 ; 1.14)$ & $0.016 \dagger \dagger$ \\
\hline Active plasma renin concentration $(\mu \mathrm{U} / \mathrm{mL})$ & 2931 & $11.35(6.2 ; 20.1)$ & 2598 & $11.1(6.1 ; 18.9)$ & 333 & $15.35(7.1 ; 36.6)$ & $<0.001+\dagger$ \\
\hline Aldosterone (ng/L) & 2931 & $38(26 ; 58)$ & 2598 & $38(26 ; 58)$ & 333 & $38(22 ; 60)$ & $0.319+\dagger$ \\
\hline Aldosterone-to-renin ratio & 2931 & $5.68(2.93 ; 10.75)$ & 2598 & $5.89(3.18 ; 11.11)$ & 333 & $3.59(1.54 ; 8.64)$ & $<0.001 \dagger \dagger$ \\
\hline Antihypertensive medication, $\mathrm{n}(\%)$ & 2931 & $909(31)$ & 2598 & $649(25)$ & 333 & $240(72)$ & $<0.001+\dagger$ \\
\hline ACE inhibitors, $n(\%)$ & 2931 & $384(13)$ & 2598 & $255(10)$ & 333 & $129(39)$ & $<0.0019$ \\
\hline Angiotensin receptor blockers, $n(\%)$ & 2931 & $223(8)$ & 2598 & $172(7)$ & 333 & $51(15)$ & $<0.001 \rrbracket$ \\
\hline Beta blockers, $\mathrm{n}(\%)$ & 2931 & $554(19)$ & 2598 & $403(15)$ & 333 & $151(45)$ & $<0.0019$ \\
\hline Diuretics, $\mathrm{n}(\%)$ & 2931 & $521(18)$ & 2598 & $365(14)$ & 333 & $156(47)$ & $<0.0019$ \\
\hline Calcium channel blockers, $n(\%)$ & 2931 & $230(8)$ & 2598 & $162(6)$ & 333 & $68(20)$ & $<0.0019$ \\
\hline
\end{tabular}

${ }^{*}$ Mean $\pm \mathrm{SD}$, median (first quartile; third quartile), or number of participants (proportion in \%).

†Normal glucose tolerance and pre-diabetes.

łKnown and newly diagnosed type 2 diabetes.

$\S$ The $p$ value is related to the null hypothesis of no difference between participants without diabetes and with type 2 diabetes.

१ा $\chi^{2}$ test.

**t-Test.

t†Mann-Whitney U test.

¥¥Participants with glucose-lowering drugs were excluded; the median $\mathrm{HbA} 1 \mathrm{c}$ of all participants with type 2 diabetes, including those using glucose-lowering medication, was $6.4 \%$ $(5.9 ; 7.0)(46.4 \mathrm{mmol} / \mathrm{mol}(41.0 ; 53.0))$.

BMI, body mass index; eGFR, estimated glomerular filtration rate; HbA1c, hemoglobin A1c; HOMA-B, homeostasis model assessment of beta cell function; HOMA-IR, homeostasis model assessment of insulin resistance.

were performed using the statistical environment $\mathrm{R}$ V.3.6.0 (R Development Core Team. R: A Language and Environment for Statistical Computing. Vienna, Austria: R Foundation for Statistical Computing; 2019).

\section{RESULTS}

\section{Study population characteristics}

Table 1 displays the baseline characteristics of the total study population and stratified by T2D. Active plasma renin concentrations were higher in participants with T2D, whereas aldosterone concentrations were similar in participants with and without T2D. The ARR was lower in participants with T2D.

Cross-sectional associations of the RAAS parameters with pre-diabetes, T2D, measures of glucose metabolism and adipokines

Renin, but not aldosterone, was significantly positively associated with prevalent pre-diabetes and T2D. The ARR was inversely associated with T2D (table 2).

Table 3 displays the associations of the RAAS parameters with continuous measures of glucose metabolism. 
Table 2 ORs $(95 \% \mathrm{Cl}$ ) for prevalent pre-diabetes (vs normal glucose tolerance) and prevalent type 2 diabetes (vs no type 2 diabetes) per SD increase of active plasma renin concentration, aldosterone and ARR

\begin{tabular}{|c|c|c|c|c|}
\hline & $\begin{array}{l}\text { Prevalent pre-diabetes (yes: } \\
\mathrm{n}=488 \text {; no: } \mathrm{n}=\mathbf{2 1 1 0} \text { ) }\end{array}$ & $P$ value & $\begin{array}{l}\text { Prevalent type two diabetes } \\
\text { (yes: } n=333 \text {; no: } n=2598 \text { ) }\end{array}$ & $P$ value \\
\hline Renin & 1.20 (1.07 to 1.35$)$ & 0.002 & 1.25 (1.10 to 1.43$)$ & $<0.001$ \\
\hline Aldosterone & 1.10 (0.98 to 1.24$)$ & 0.095 & $1.04(0.91$ to 1.19$)$ & 0.547 \\
\hline ARR & 0.92 (0.82 to 1.03$)$ & 0.151 & $0.82(0.72$ to 0.93$)$ & 0.002 \\
\hline
\end{tabular}

Bold print indicates significance after correction for multiple testing using the Bonferroni method $(p<0.008(0.05 \div 6))$.

The logistic regression models were adjusted for sex, age, BMI, arterial hypertension, eGFR, potassium, use of ACE inhibitors, angiotensin receptor blockers, beta blockers, diuretics and calcium channel blockers.

ARR, aldosterone-to-renin ratio; BMI, body mass index; eGFR, estimated glomerular filtration rate.

After Bonferroni correction for multiple testing, renin was positively associated with fasting glucose, 2-hour glucose, insulin, proinsulin, HOMA-B and HOMA-IR. In a sensitivity analysis, the associations of renin with insulin and HOMA-IR were additionally adjusted for fasting glucose and remained significant $(\beta$ : $0.11 \pm 0.02$ and $0.09 \pm 0.02$, respectively; $\mathrm{p}<0.001$ for both). Aldosterone was positively associated with insulin, proinsulin and HOMA-IR, whereas the ARR was inversely associated with fasting glucose, insulin and HOMA-IR. After correction for multiple testing, no significant associations of the RAAS parameters with HbAlc and the proinsulin-toinsulin ratio were found.

The associations of the RAAS parameters with adipokines are presented in table 4. After correction for multiple testing, renin was positively associated with leptin and retinol-binding protein-4, and aldosterone was positively associated with leptin. The ARR was not significantly associated with any adipokine.

\section{Longitudinal analyses of the association of the RAAS} parameters with pre-diabetes, T2D and continuous measures of glucose metabolism

None of the RAAS parameters was significantly associated with incident pre-diabetes and T2D (table 5 and online supplemental table 4). Online supplemental tables 5 and 6 show the longitudinal associations of the RAAS parameters with deltas of continuous measures of glucose metabolism. After correction for multiple testing, renin was significantly inversely associated with changes in insulin, and the ARR was positively associated with changes in insulin. No other significant associations of the RAAS parameters with changes of continuous measures of glucose metabolism were observed after correction for multiple testing.

The results after exclusion of participants taking ACE inhibitors, angiotensin receptor blockers, beta blockers, diuretics and calcium channel blockers were comparable (online supplemental tables 1-4 and 6).

\section{DISCUSSION}

Renin was associated with prevalent but not with incident pre-diabetes and T2D in the population-based KORA F4/ FF4 cohort. In line, renin and aldosterone were associated with continuous measures of glucose metabolism in the cross-sectional analysis but not with changes of these traits during follow-up. Of note, none of the associations of aldosterone with measures of glucose metabolism was independent of renin. In fact, the ARR was even inversely

Table 3 Cross-sectional beta estimates \pm SE of the associations of active plasma renin concentration, aldosterone and ARR (per SD) and continuous measures of glucose metabolism (per SD)

\begin{tabular}{|c|c|c|c|c|}
\hline & $\mathbf{N}$ & Renin & Aldosterone & ARR \\
\hline Fasting glucose & 2764 & $0.08 \pm 0.02^{\star \star *}$ & $0.01 \pm 0.02$ & $-0.07 \pm 0.02^{\star \star \star}$ \\
\hline 2-hour glucose & 2710 & $0.08 \pm 0.02^{\star \star \star}$ & $0.02 \pm 0.02$ & $-0.06 \pm 0.02^{\star \star}$ \\
\hline $\mathrm{HbA1c}$ & 2764 & $0.05 \pm 0.02^{\star \star}$ & $-0.01 \pm 0.02$ & $-0.05 \pm 0.02^{\star \star}$ \\
\hline Insulin & 2743 & $0.13 \pm 0.02^{\star \star \star}$ & $0.06 \pm 0.02^{\star \star \star}$ & $-0.07 \pm 0.02^{\star \star *}$ \\
\hline Proinsulin & 1438 & $0.11 \pm 0.03^{* \star *}$ & $0.08 \pm 0.02^{\star * *}$ & $-0.05 \pm 0.03^{*}$ \\
\hline Proinsulin-to-insulin ratio & 1438 & $0.01 \pm 0.03$ & $0.06 \pm 0.03^{*}$ & $0.02 \pm 0.03$ \\
\hline HOMA-B & 2743 & $0.09 \pm 0.02^{* * *}$ & $0.05 \pm 0.02^{\star \star}$ & $-0.04 \pm 0.02^{*}$ \\
\hline HOMA-IR & 2743 & $0.13 \pm 0.02^{\star * *}$ & $0.06 \pm 0.02^{\star * *}$ & $-0.08 \pm 0.02^{\star \star *}$ \\
\hline
\end{tabular}

${ }^{*} \mathrm{P}<0.05 ;{ }^{* *} \mathrm{p}<0.01 ;{ }^{* * *} \mathrm{p}<0.001$; bold print indicates significance after correction for multiple testing using the Bonferroni method ( $\mathrm{p}<0.0021$ $(0.05 \div 24))$.

The linear regression models were adjusted for sex, age, BMI, arterial hypertension, eGFR, potassium, use of ACE inhibitors, angiotensin receptor blockers, beta blockers, diuretics and calcium channel blockers.

ARR, aldosterone-to-renin ratio; BMI, body mass index; eGFR, estimated glomerular filtration rate; HbA1c, hemoglobin A1c; HOMA-B, homeostasis model assessment of beta cell function; HOMA-IR, homeostasis model assessment of insulin resistance. 
Table 4 Cross-sectional beta estimates \pm SE of the associations of active plasma renin concentration, aldosterone and ARR (per SD) and adipokines (per SD).

\begin{tabular}{llccc}
\hline & $\mathbf{N}$ & Renin & Aldosterone & ARR \\
\hline Leptin & 2922 & $\mathbf{0 . 0 5} \pm \mathbf{0 . 0 1 ^ { * * * }}$ & $\mathbf{0 . 0 5} \pm \mathbf{0 . 0 1 ^ { * * * }}$ & $-0.01 \pm 0.01$ \\
Retinol-binding protein-4 & 2922 & $\mathbf{0 . 0 7} \pm \mathbf{0 . 0 2 ^ { * * * }}$ & $0.04 \pm 0.02^{*}$ & $-0.03 \pm 0.02$ \\
Adiponectin & 1093 & $-0.07 \pm 0.03^{*}$ & $-0.05 \pm 0.03$ & $0.03 \pm 0.03$ \\
Omentin-1 & 1093 & $-0.01 \pm 0.04$ & $0.00 \pm 0.03$ & $-0.001 \pm 0.03$ \\
Chemerin & 1702 & $0.04 \pm 0.03$ & $0.02 \pm 0.02$ & $-0.02 \pm 0.03$ \\
Progranulin & 1702 & $-0.03 \pm 0.03$ & $-0.01 \pm 0.03$ & $0.02 \pm 0.03$ \\
Vaspin & 1702 & $-0.01 \pm 0.03$ & $0.07 \pm 0.02^{\star \star}$ & $0.06 \pm 0.03^{\star}$ \\
\hline
\end{tabular}

${ }^{*} \mathrm{P}<0.05 ;{ }^{* *} \mathrm{p}<0.01 ;{ }^{* \star *} \mathrm{p}<0.001$; bold print indicates significance after correction for multiple testing using the Bonferroni method ( $\mathrm{p}<0.0024$ $(0.05 \div 21))$.

The linear regression models were adjusted for sex, age, BMI, arterial hypertension, eGFR, potassium, use of ACE inhibitors, angiotensin receptor blockers, beta blockers, diuretics and calcium channel blockers.

ARR, aldosterone-to-renin-ratio; BMI, body mass index; eGFR, estimated glomerular filtration rate.

associated with prevalent T2D and with fasting glucose, insulin and HOMA-IR. One possible explanation for the association of the RAAS parameters with prevalent diabetes might be hyperglycemia-induced RAAS activation. Indeed, renin was associated with fasting glucose and 2-hour glucose. However, although significant, these associations were moderate and do not fully explain the stronger associations of renin with insulin and insulin resistance, which remained significant after adjustment for fasting glucose.

Our findings indicate that the independent associations between aldosterone and alterations of glucose tolerance observed in primary aldosteronism ${ }^{8-15}$ are not readily transferable to a general population. In primary aldosteronism and in most preclinical studies, aldosterone values are considerably higher than in the general population. Furthermore, the association of primary aldosteronism with diabetes may partly be explained by concomitant subclinical hypercortisolism. ${ }^{34}$

However, our data are also not fully consistent with some previous population-based studies. In a Japanese population, baseline plasma aldosterone was associated with the development of insulin resistance during follow-up. ${ }^{22}$ Renin was not measured in this study; thus, the dependence on RAAS activation could not be determined. Furthermore, since only 14 participants developed diabetes during follow-up, an analysis of incident diabetes was not possible. In line with our data, HbA1c and fasting glucose were not associated with aldosterone at baseline. ${ }^{22}$ A positive association of both aldosterone and renin with incident diabetes was observed in African-Americans, ${ }^{23}$ and an association of aldosterone, but not renin, with incident diabetes was shown in Chinese and African-Americans, in whom the association was significantly stronger than in Hispanic and nonHispanic white Americans. ${ }^{24}$ These data suggest ethnic variations in the associations of the RAAS with glucose metabolism. Importantly, ethnic differences have been observed for other RAAS effects. For instance, blacks have a higher sensitivity of blood pressure to aldosterone than whites. ${ }^{35}$

Our study shows some discrepancies between crosssectional and longitudinal results. There are several possible reasons for the observation of significant positive associations of renin with type 2 diabetes and continuous measures of glucose metabolism cross-sectionally but not longitudinally. Reverse causation, for example, activation of the RAAS by hyperglycemia and/or other associated factors, may be one explanation for the associations of renin with type 2 diabetes and measures of glucose metabolism in the cross-sectional analyses, which do not translate into longitudinal associations.

Table 5 ORs $(95 \% \mathrm{Cl}$ ) for incident pre-diabetes/type 2 diabetes (vs non-progressors to pre-diabetes/type 2 diabetes from normal glucose tolerance) and incident type 2 diabetes (vs non-progressors to type 2 diabetes from normal glucose tolerance or pre-diabetes) per SD increase of active plasma renin concentration, aldosterone and ARR

\begin{tabular}{lllll}
\hline & $\begin{array}{l}\text { Incident pre-diabetes/type 2 diabetes } \\
\text { (yes: } \mathbf{n = 2 8 3} \text {; } \mathbf{n o :} \mathbf{n = 1 2 6 0 )}\end{array}$ & $\mathbf{P}$ value & $\begin{array}{l}\text { Incident type 2 diabetes (yes: } \\
\mathbf{n}=\mathbf{1 3 0} ; \mathbf{n o :} \mathbf{n = 1 7 0 4 )}\end{array}$ & $\mathbf{P}$ value \\
\hline Renin & $1.05(0.9-1.22)$ & 0.538 & $1.12(0.92-1.36)$ & 0.257 \\
Aldosterone & $1.00(0.86-1.16)$ & 0.993 & $0.91(0.74-1.11)$ & 0.343 \\
ARR & $0.97(0.83-1.13)$ & 0.660 & $0.87(0.71-1.05)$ & 0.152 \\
\hline
\end{tabular}

Significance level after correction for multiple testing using the Bonferroni method $(p<0.008(0.05 \div 6)$ ).

The logistic regression models were adjusted for sex, age, BMI, arterial hypertension, eGFR, potassium, use of ACE inhibitors, angiotensin receptor blockers, beta blockers, diuretics and calcium channel blockers.

ARR, aldosterone-to-renin ratio; BMI, body mass index; eGFR, estimated glomerular filtration rate. 
Another possibility is residual confounding, which might occur despite careful adjustments of the analyses. Furthermore, a lack of power might have resulted in the non-significance of the association of renin with incident diabetes, since the number of incident type 2 diabetes cases was lower than the number of prevalent cases. Renin showed a trend towards a positive association with incident type 2 diabetes. The detection of a significant association in a larger study population cannot be excluded. However, aldosterone was neither cross-sectionally nor longitudinally associated with type 2 diabetes and the cross-sectional associations of aldosterone with insulin, proinsulin and HOMA-IR were not independent of renin. The OR for the association of aldosterone with incident type 2 diabetes was $<1.0$, strongly arguing against a significant positive association, which might have been detectable in a larger study population.

Renin and aldosterone were associated with leptin, a finding in line with preclinical data showing that leptin might mediate aldosteronism in obesity. Previous data suggest a partially renin-independent relationship of aldosterone and leptin. Adrenocortical cells were demonstrated to coexpress CYP11B2 and leptin receptors. ${ }^{36}$ Leptin directly induced aldosterone secretion in a preclinical model ${ }^{36}$ and mediated hypertension via aldosterone-dependent mechanisms in female mice. ${ }^{37}$ In addition to the direct relation to aldosterone, leptin also induces sympatho-excitatory effects and subsequent activation of the RAAS. ${ }^{38}$ In our study, the association of aldosterone with leptin was not independent of renin (the ARR was not associated with leptin), suggesting a RAAS-dependency of leptin rather than a direct interaction with aldosterone in the general population.

Renin was further associated with retinol-binding protein- 4 but not with the other assessed adipokines. Adiponectin was inversely associated with renin, but significance was not maintained after correction for multiple testing. Previously, influences on adiponectin expression and secretion were suggested to link aldosterone to the metabolic syndrome. In vitro, aldosterone perturbs adiponectin expression in 3T3-L1 adipocytes. ${ }^{39}$ However, the influence of aldosterone on 3T3-L1 adipocytes appeared to be mediated mainly via the glucocorticoid receptor and thus required relatively high aldosterone concentrations. ${ }^{39}$ In line, adiponectin levels were lower in patients with primary aldosteronism compared with patients with essential hypertension. ${ }^{40}$ However, adiponectin and leptin expression was not increased in visceral adipose tissue of patients with aldosterone-producing adenoma. ${ }^{41}$ Our data indicate no major role for RAAS parameters for adiponectin concentrations in a general population. Also, the other investigated adipokines omentin-1, chemerin, progranulin and vaspin were not significantly related to renin or aldosterone. Thus, a wide interaction of the RAAS with adipose tissue secretory products in a general population is not suggested by these data.

\section{Strengths and limitations}

The findings of our study are based on a large and well-characterized prospective community-based study. Glucose tolerance status was assessed by oral glucose tolerance test at baseline and follow-up. Several further parameters for the characterization of glucose metabolism and measurements of both renin and aldosterone as well as of a number of adipokines were available. As the KORA study included nearly exclusively Caucasians, our results may not apply to other ethnic groups, and the study is not suitable to directly investigate the question of racial differences of RAAS effects. Many of the participants with diabetes used antihypertensive and/or diuretic medication. Although adjustment was performed, residual confounding cannot be excluded. However, the results after exclusion of participants on potentially confounding medication were similar. Proinsulin and some of the adipokines were available only in subgroups, limiting the generalizability and power of the concerned analyses. Renin and aldosterone were measured at baseline only, precluding the investigation of possible changes over time. No detailed data on salt intake or urinary sodium excretion were available. Dietary salt intake may affect glucose metabolism via RAAS and/or other parameters, although data are conflicting and the mechanisms remain unclear. In one study, dietary sodium restriction resulted in a decreased insulin sensitivity in participants with type 2 diabetes ${ }^{42}$ whereas in another study including non-diabetic participants, sodium restriction did not affect insulin sensitivity but impaired insulin secretion. ${ }^{43}$ However, higher sodium excretion was associated with a higher risk of type 2 diabetes in a meta-analysis of observational studies. ${ }^{44}$ Therefore, RAAS-dependent and independent effects of dietary sodium on glucose metabolism cannot be excluded.

\section{CONCLUSION}

At baseline, renin was associated with prevalent prediabetes, T2D and continuous surrogate parameters of IGT and insulin sensitivity. Aldosterone was associated with insulin and HOMA-IR, whereas the ARR was inversely associated with prevalent T2D, fasting glucose, insulin and HOMA-IR, suggesting that aldosterone is associated with insulin resistance through the activation of the RAAS in a general Western European population. Renin, aldosterone and the ARR were not associated with incident T2D or changes in continuous measures of glucose metabolism.

\section{Author affiliations}

${ }^{1}$ Medizinische Klinik und Poliklinik IV, Klinikum der Universität München, Munich, Germany

${ }^{2}$ German Center for Diabetes Research, Neuherberg, Germany

${ }^{3}$ German Center for Diabetes Research, Düsseldorf, Germany

${ }^{4}$ Department of Endocrinology and Diabetology, Medical Faculty and University Hospital Düsseldorf, Heinrich-Heine-University, Düsseldorf, Germany

${ }^{5}$ Institute of Clinical Diabetology, German Diabetes Center, Leibniz Center for

Diabetes Research at Heinrich-Heine-University, Düsseldorf, Germany

${ }^{6}$ Freie Waldorfschule Augsburg, Augsburg, Germany 
${ }^{7}$ Institute of Epidemiology, Helmholtz Zentrum München - German Research Center for Environmental Health (GmbH), Neuherberg, Germany

${ }^{8}$ Institute for Medical Information Processing, Biometry, and Epidemiology, Pettenkofer School of Public Health, LMU München, Munich, Germany ${ }^{9}$ KORA Study Centre, University Hospital Augsburg, Augsburg, Germany ${ }^{10}$ Chair of Epidemiology, University Hospital Augsburg, Augsburg, Germany ${ }^{11}$ Independent Research Group Clinical Epidemiology, Helmholtz Zentrum München Deutsches Forschungszentrum für Gesundheit und Umwelt, Neuherberg, Germany ${ }^{12}$ DZHK (German Centre for Cardiovascular Research), partner site Munich Heart Alliance, Munich, Germany

${ }^{13}$ Institute of Epidemiology and Medical Biometry, Ulm University, Ulm, Germany ${ }^{14}$ Deutsches Herzzentrum München, Technische Universität München, Munich, Germany

${ }^{15}$ German Diabetes Center, Leibniz Institute at Heinrich Heine University Düsseldorf, Institute of Biometrics and Epidemiology, Düsseldorf, Germany

${ }^{16}$ Department of Medicine, University of Leipzig, Leipzig, Germany

${ }^{17}$ Institute of Human Genetics, Technische Universität München, Munchen, Germany

Acknowledgements We gratefully acknowledge the contribution of all field staff members conducting the KORA F4 study and thank all study participants.

Contributors Conception and design of the study: MRe, BT, CM, CH, MH, AP, WK and WR; collection of data: $\mathrm{BT}$, MRe, MB, CT, CM, CH, MH, AP, WK, WR, MRo, MS and TM; data analysis, interpretation of results and writing of the manuscript: $C T$, $\mathrm{KR}, \mathrm{CS}, \mathrm{HM}, \mathrm{HT}, \mathrm{BT}, \mathrm{CH}$ and JS; all authors revised the manuscript critically for important intellectual content and approved the final version. Guarantor: CT.

Funding The study was supported by a research grant from the Virtual Diabetes Institute (Helmholtz Zentrum München) and the Clinical Cooperation Group Diabetes, Ludwig-Maximilians-University München and Helmholtz Zentrum München, and by the German Diabetes Center. The German Diabetes Center was supported by the Federal Ministry of Health (Berlin, Germany) and the Ministry of Culture and Science of the state North Rhine Westphalia (Düsseldorf, Germany). The KORA study was initiated and financed by the Helmholtz Zentrum München German Research Center for Environmental Health, which is funded by the German Federal Ministry of Education and Research (BMBF) and by the State of Bavaria. This study was also supported by grants from the German Federal Ministry of Education and Research (BMBF) to the German Center for Diabetes Research e.V. (DZD). Further support was obtained from the Deutsche Diabetes Gesellschaft (DDG) and from the German Research Foundation (DFG, grant RA-45913/3-1). MRe received funding by the Else Kröner-Fresenius Stiftung (2012_A103, 2015_A228, and 2019_A104; Else-Kröner Hyperaldosteronismus - German Conn Registry), by the European Research Council under the European Union's Horizon 2020 research and innovation programme (grant agreement № 694913 to M. Reincke), and by the DFG (CRC/TRR 205 'The Adrenal Gland').

Disclaimer The funding sources had no role in in study design; in the collection, analysis and interpretation of data; in the writing of the report; and in the decision to submit the article for publication.

Competing interests $\mathrm{CH}$ received a research grant from Sanofi-Aventis. MRo reports consulting fees from Eli Lilly, Terra Firma, Sanofi US, Fishawack Group, Target Pharmasolutions, Bristol-Myers Squibb and Inventiva, honoraria for lectures from Boehringer-Ingelheim Pharma, Sanofi US, Novo Nordisk, payment for expert testimony from Novartis and Boehringer Ingelheim, participation on a Data Safety Monitoring Board or Advisory Board: Poxel, Servier. The reported disclosures are not directly related to this manuscript. The other authors declare that they have no conflict of interest associated with this manuscript. The results presented in this paper have not been published previously in whole or part.

Patient consent for publication Not applicable.

Ethics approval This study involves human participants and was approved by Ethics Committees of the Bavarian Medical Association (approval number 06068). Participants gave informed consent to participate in the study before taking part.

Provenance and peer review Not commissioned; externally peer reviewed.

Data availability statement Data are available on reasonable request. The data are subject to national data protection laws and restrictions were imposed by the Ethics Committee of the Bavarian Chamber of Physicians to ensure data privacy of the study participants. Therefore, data cannot be made freely available in a public repository. However, data can be requested through an individual project agreement with KORA via the online portal KORA.passt (https://epi.helmholtzmuenchen.de/).
Supplemental material This content has been supplied by the author(s). It has not been vetted by BMJ Publishing Group Limited (BMJ) and may not have been peer-reviewed. Any opinions or recommendations discussed are solely those of the author(s) and are not endorsed by BMJ. BMJ disclaims all liability and responsibility arising from any reliance placed on the content. Where the content includes any translated material, BMJ does not warrant the accuracy and reliability of the translations (including but not limited to local regulations, clinical guidelines, terminology, drug names and drug dosages), and is not responsible for any error and/or omissions arising from translation and adaptation or otherwise.

Open access This is an open access article distributed in accordance with the Creative Commons Attribution Non Commercial (CC BY-NC 4.0) license, which permits others to distribute, remix, adapt, build upon this work non-commercially, and license their derivative works on different terms, provided the original work is properly cited, appropriate credit is given, any changes made indicated, and the use is non-commercial. See: http://creativecommons.org/licenses/by-nc/4.0/.

\section{ORCID iDs}

Cornelia Then http://orcid.org/0000-0001-5180-1973

Christian Herder http://orcid.org/0000-0002-2050-093X

Barbara Thorand http://orcid.org/0000-0002-8416-6440

\section{REFERENCES}

1 Ehrhart-Bornstein M, Lamounier-Zepter V, Schraven A, et al. Human adipocytes secrete mineralocorticoid-releasing factors. Proc Natl Acad Sci U S A 2003;100:14211-6.

2 Ohno Y, Sone M, Inagaki N, et al. Obesity as a key factor underlying idiopathic hyperaldosteronism. J Clin Endocrinol Metab 2018; 103:4456-64.

3 Thuzar M, Stowasser M. The mineralocorticoid receptor-an emerging player in metabolic syndrome? J Hum Hypertens 2021;35:117-23.

4 Jin N, Wang Y, Liu L, et al. Dysregulation of the renin-angiotensin system and cardiometabolic status in mice fed a long-term high-fat diet. Med Sci Monit 2019;25:6605-14.

5 Luther JM, Luo P, Kreger MT, et al. Aldosterone decreases glucosestimulated insulin secretion in vivo in mice and in murine islets. Diabetologia 2011;54:2152-63.

6 Selvaraj J, Sathish S, Mayilvanan C, et al. Excess aldosteroneinduced changes in insulin signaling molecules and glucose oxidation in gastrocnemius muscle of adult male rat. $\mathrm{Mol}$ Cell Biochem 2013;372:113-26.

7 Jia G, Lockette W, Sowers JR. Mineralocorticoid receptors in the pathogenesis of insulin resistance and related disorders: from basic studies to clinical disease. Am J Physiol Regul Integr Comp Physiol 2021;320:R276-86.

8 Bothou C, Beuschlein F, Spyroglou A. Links between aldosterone excess and metabolic complications: a comprehensive review. Diabetes Metab 2020;46:1-7.

9 Liu Y, Zhou L, Liu Z, et al. Higher blood urea nitrogen and urinary calcium: new risk factors for diabetes mellitus in primary aldosteronism patients. Front Endocrinol 2020;11:23.

10 Chang $\mathrm{C}-\mathrm{H}$, Hu Y-H, Huang $\mathrm{K}-\mathrm{H}$, et al. Higher screening aldosterone to renin ratio in primary aldosteronism patients with diabetes mellitus. J Clin Med 2018;7:360.

11 Giacchetti G, Ronconi V, Turchi F, et al. Aldosterone as a key mediator of the cardiometabolic syndrome in primary aldosteronism: an observational study. J Hypertens 2007;25:177-86.

12 Catena C, Lapenna R, Baroselli S, et al. Insulin sensitivity in patients with primary aldosteronism: a follow-up study. J Clin Endocrinol Metab 2006;91:3457-63.

13 Sindelka G, Widimský J, Haas T, et al. Insulin action in primary hyperaldosteronism before and after surgical or pharmacological treatment. Exp Clin Endocrinol Diabetes 2000;108:21-5.

14 Fischer E, Adolf C, Pallauf A, et al. Aldosterone excess impairs first phase insulin secretion in primary aldosteronism. J Clin Endocrinol Metab 2013;98:2513-20.

15 Adler GK, Murray GR, Turcu AF, et al. Primary aldosteronism decreases insulin secretion and increases insulin clearance in humans. Hypertension 2020;75:1251-9.

16 Freel EM, Tsorlalis IK, Lewsey JD, et al. Aldosterone status associated with insulin resistance in patients with heart failure--data from the ALOFT study. Heart 2009;95:1920-4.

17 Srinivasa S, Fitch KV, Wong K, et al. RAAS activation is associated with visceral adiposity and insulin resistance among HIV-infected patients. J Clin Endocrinol Metab 2015;100:2873-82.

18 Murphy CA, Fitch KV, Feldpausch M, et al. Excessive adiposity and metabolic dysfunction relate to reduced natriuretic peptide during RAAS activation in HIV. J Clin Endocrinol Metab 2018;103:1558-65. 
19 Garg R, Hurwitz S, Williams GH, et al. Aldosterone production and insulin resistance in healthy adults. J Clin Endocrinol Metab 2010;95:1986-90.

20 Hannemann A, Meisinger C, Bidlingmaier M, et al. Association of plasma aldosterone with the metabolic syndrome in two German populations. Eur J Endocrinol 2011;164:751-8.

21 Kidambi S, Kotchen JM, Krishnaswami S, et al. Hypertension, insulin resistance, and aldosterone: sex-specific relationships. J Clin Hypertens 2009;11:130-7.

22 Kumagai E, Adachi $\mathrm{H}$, Jacobs DR, et al. Plasma aldosterone levels and development of insulin resistance: prospective study in a general population. Hypertension 2011;58:1043-8.

23 Joseph JJ, Echouffo-Tcheugui JB, Kalyani RR, et al. Aldosterone, renin, and diabetes mellitus in African Americans: the Jackson heart study. J Clin Endocrinol Metab 2016;101:1770-8.

24 Joseph JJ, Echouffo Tcheugui JB, Effoe VS, et al. Renin-angiotensinaldosterone system, glucose metabolism and incident type 2 diabetes mellitus: MESA. J Am Heart Assoc 2018;7:e009890.

25 Rathmann W, Strassburger K, Heier M, et al. Incidence of type 2 diabetes in the elderly German population and the effect of clinical and lifestyle risk factors: KORA S4/F4 cohort study. Diabet Med 2009;26:1212-9.

26 Huth C, von Toerne C, Schederecker F, et al. Protein markers and risk of type 2 diabetes and prediabetes: a targeted proteomics approach in the KORA F4/FF4 study. Eur $\mathrm{J}$ Epidemiol 2019;34:409-22.

27 Then C, Gar C, Thorand B, et al. Proinsulin to insulin ratio is associated with incident type 2 diabetes but not with vascular complications in the KORA F4/FF4 study. BMJ Open Diabetes Res Care 2020;8:e001425.

28 Manolopoulou J, Bielohuby M, Caton SJ, et al. A highly sensitive immunofluorometric assay for the measurement of aldosterone in small sample volumes: validation in mouse serum. $J$ Endocrinol 2008:196:215-24

29 Sujana C, Seissler J, Jordan J, et al. Associations of cardiac stress biomarkers with incident type 2 diabetes and changes in glucose metabolism: KORA F4/FF4 study. Cardiovasc Diabetol 2020;19:178.

30 Ebert T, Gebhardt C, Scholz M, et al. Relationship between 12 adipocytokines and distinct components of the metabolic syndrome. $J$ Clin Endocrinol Metab 2018;103:1015-23.

31 Wolf K, Popp A, Schneider A, et al. Association between longterm exposure to air pollution and biomarkers related to insulin resistance, subclinical inflammation, and adipokines. Diabetes 2016;65:3314-26.
32 Herder C, Kannenberg JM, Niersmann C, et al. Independent and opposite associations of serum levels of omentin-1 and adiponectin with increases of glycaemia and incident type 2 diabetes in an older population: KORA F4/FF4 study. Eur $J$ Endocrinol 2017;177:277-86.

33 Levey AS, Stevens LA, Schmid CH, et al. A new equation to estimate glomerular filtration rate. Ann Intern Med 2009;150:604-12.

34 Akehi Y, Yanase T, Motonaga R, et al. High prevalence of diabetes in patients with primary aldosteronism (PA) associated with subclinical hypercortisolism and prediabetes more prevalent in bilateral than unilateral PA: a large, multicenter cohort study in Japan. Diabetes Care 2019;42:938-45.

35 Tu W, Eckert GJ, Hannon TS, et al. Racial differences in sensitivity of blood pressure to aldosterone. Hypertension 2014;63:1212-8.

36 Huby A-C, Antonova G, Groenendyk J, et al. Adipocyte-derived hormone leptin is a direct regulator of aldosterone secretion, which promotes endothelial dysfunction and cardiac fibrosis. Circulation 2015;132:2134-45.

37 Huby A-C, Otvos L, Belin de Chantemèle EJ. Leptin induces hypertension and endothelial dysfunction via aldosteronedependent mechanisms in obese female mice. Hypertension 2016;67:1020-8

38 Kalil GZ, Haynes WG. Sympathetic nervous system in obesityrelated hypertension: mechanisms and clinical implications. Hypertens Res 2012;35:4-16.

39 Li P, Zhang X-N, Pan C-M, et al. Aldosterone perturbs adiponectin and PAI-1 expression and secretion in 3T3-L1 adipocytes. Horm Metab Res 2011;43:464-9.

40 Fallo F, Della Mea P, Sonino N, et al. Adiponectin and insulin sensitivity in primary aldosteronism. Am J Hypertens 2007;20:855-61.

41 Urbanet R, Pilon C, Calcagno A, et al. Analysis of insulin sensitivity in adipose tissue of patients with primary aldosteronism. J Clin Endocrinol Metab 2010:95:4037-42.

42 Petrie JR, Morris AD, Minamisawa K, et al. Dietary sodium restriction impairs insulin sensitivity in noninsulin-dependent diabetes mellitus. $J$ Clin Endocrinol Metab 1998;83:1552-7.

43 Luther JM, Byrne LM, Yu C, et al. Dietary sodium restriction decreases insulin secretion without affecting insulin sensitivity in humans. J Clin Endocrinol Metab 2014:99:E1895-902.

44 Kolahdouz-Mohammadi R, Soltani S, Clayton ZS, et al. Sodium status is associated with type 2 diabetes mellitus: a systematic review and meta-analysis of observational studies. Eur J Nutr 2021;60:3543-65. 